\title{
Traffic Organization and Control Strategy of Expressway Tunnel Group
}

\author{
Xuan $\mathrm{Mu}^{1, \mathrm{a}}$, Yunlai $\mathrm{He}^{2, \mathrm{~b}}$,Xiaoyan $\mathrm{Shi}^{2, \mathrm{c}}$, and Zhongjie Zhao ${ }^{2, \mathrm{~d}}$ \\ ${ }^{1}$ School of Shanxi, Chang'an University, Xi'an 710018, China; \\ ${ }^{2}$ School of Shanxi, Chang'an University, Xi'an 710018, China. \\ a997826142@qq.com, bheyunlai1991@163.com, 'c414711977@qq.com, d1274439507@qq.com
}

Keywords: Expressway tunnel group, Traffic organization, Control strategy.

\begin{abstract}
Expressway tunnel group compared with the independent tunnel, the operation and management is more complicated. When the tunnel had traffic accidents, if the leading scheme is not scientific, it's easy to cause serious consequences. This paper based on the actual operation's tunnel group as the background, through analyzing the traffic flow characteristics, division the monomer tunnel of the prone or multiple tunnel group into control section and the whole tunnel group into control unit, make the monomer tunnel and the different areas of tunnel group's traffic organization and the control strategy. On this basis, working out tunnel linkage control strategy, thus realize the linkage control of tunnel group section.
\end{abstract}

\section{Introduction}

All The construction of expressway tunnel in our country starts late, but developing fast, as the increasing of the proportion of tunnel in the entire line, forming the expressway tunnel group characteristics. By 2013, the National Expressway Tunnel has reached 11,359, 9606000 meters, where super-long railway tunnel 562, 2506900 meters, long tunnel 2303, 3936200 meters ${ }^{[1]}$.

Expressway tunnel group, which is a general term of two or more tunnels that distributed on the expressway section ${ }^{[2]}$. When there is a traffic accident occurs, the accident spot will form the bottleneck of the road, we must consider the interaction between the tunnels ${ }^{[3-4]}$.This paper based on the Xiaoshan tunnel and Sipo to Lingnan tunnel group as the background, which belongs to Henan Province Sanmenxia City to Xichuan County expressway's Lingbao to Lushi section, making the monomer tunnel and the different areas of tunnel group's traffic organization and the control strategy.

\section{Characteristic analysis of traffic flow operation}

The traffic flow of expressway tunnel group is mainly influenced by the proportion of the various types of vehicles, the traffic volume change and the driving speed. Through the investigation, statistics and analysis of the sanxi expressway's tunnel group which from lingbao to lushi section, the passenger cars' proportion is the largest, about 40\%; the day-hour traffic volume changes in 9:00 to 11:00 and 15:00 to 17:00 pm presentation two peak periods. By the congestion length prediction model to calculate the length of traffic jams range from $52.45 \mathrm{~m}$ to $3021.56 \mathrm{~m}$. Due to the influence of the slope and curvature factors ect of the different sections, resulting in the driving speed has a great difference between the various types of vehicles. In Sipo to Lingnan section, Sipo at downhill slope, small buses and small trucks daily average speed reached almost $90 \mathrm{~km} / \mathrm{h}$, while the Lingnan is uphill, small buses and small trucks daily average speed are not reached $70 \mathrm{~km} / \mathrm{h}$.

Through analysis the traffic flow characteristics and the traffic jam length range, division the monomer tunnel of the prone or multiple tunnel group into control sectio and the whole tunnel group into control unit, it provides a basis to making the monomer tunnel and the different areas of tunnel group's traffic organization and the control strategy. 


\section{Monomer tunnel traffic organization and control strategy}

\subsection{Control section division}

When monomer tunnel has traffic accidents, we should division the tunnel into section, and then according to the accident different classification and location to discuss the specific traffic organization and control strategy. Xiaoshan tunnel length of $2958 \mathrm{~m}$, which has three pedestrian crosswalk and two transverse traffic tubes, we divide the left and right tunnel into three sections in the transverse traffic tubes as the boundary, with the entrance proximity segment outside the cave, the whole tunnel can be divided into eight sections, as shown in Figure 1:

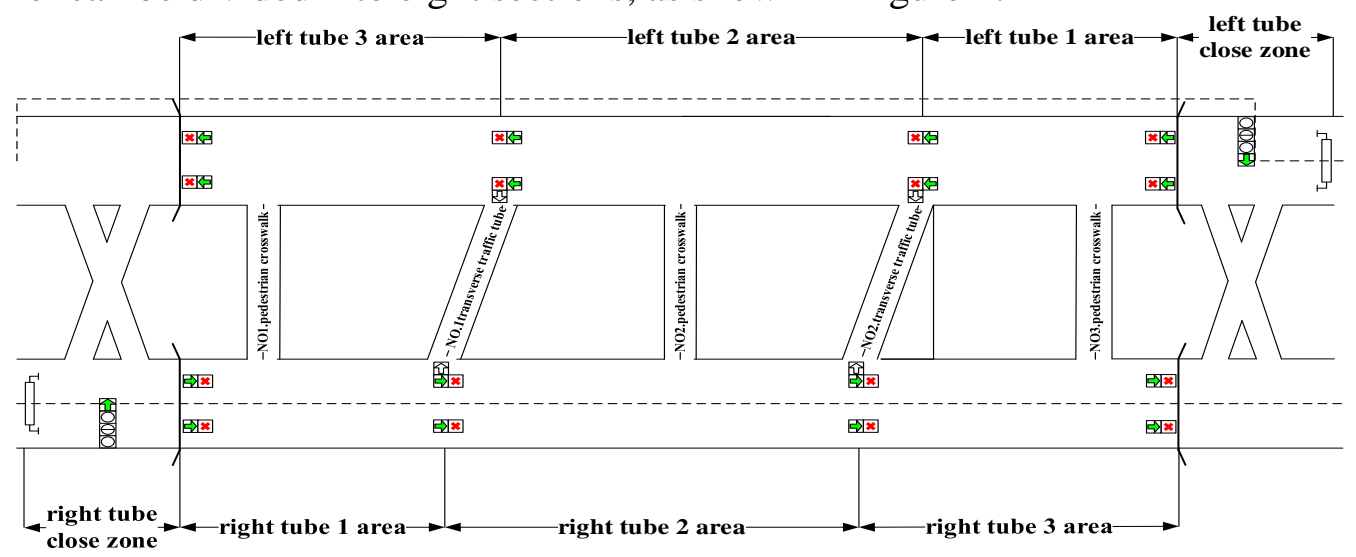

Fig. 1 Tunnel section division

\subsection{Traffic organization and control strategy}

There are often two ways of the monomer tunnel control strategy, including lane use control and area control. When each section of Xiaoshan right hole tunnel occurred single point of mild and serious traffic accident, and minor accident occurred in the outside lane, just blocking outside lane, serious accident blocking two-lane. Take the right hole 1section as example, the traffic organization and the control strategy are shown in figure 2 to figure 3.

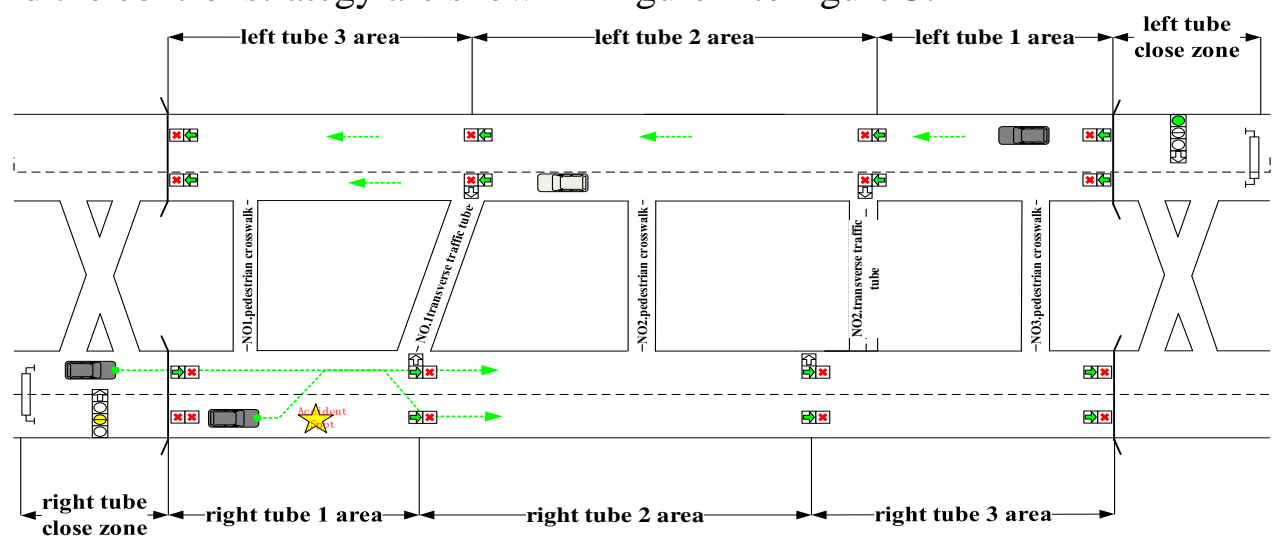

Fig. 2 Section 1 of Xiaoshan tunnel right hole minor incident traffic organization and control strategy

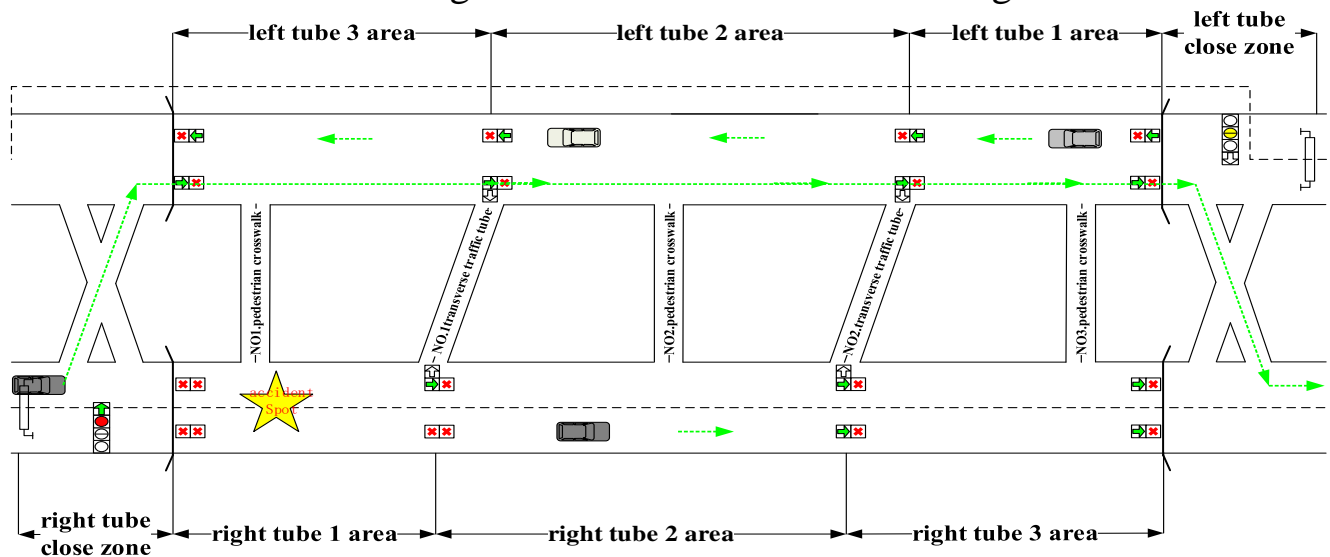


Fig. 3 Section 1 of Xiaoshan tunnel right hole serious accident traffic organization and control strategy

\section{Tunnel group section traffic organization and control strategy}

\subsection{Control unit division}

In each section of the tunnel group, along the driving direction divide the tunnel, between tunnel and tunnel sections into a control unit. The distribution of Sipo to lingnan tunnel group is dense, the control unit of the mutual linkage is divided into the following table1.

Table1 Control unit division

\begin{tabular}{|c|c|c|}
\hline NO. & The control unit (right line) & The control unit (left line) \\
\hline 1 & $\begin{array}{l}\text { sanmenxia --lingbao east interworking } \\
\text { section (unit 1) }\end{array}$ & $\begin{array}{l}\text { lingbao east interworking --sanmenxia } \\
\text { section (unit 2)) }\end{array}$ \\
\hline 2 & $\begin{array}{l}\text { lingbao east interworking -- sipo section } \\
\text { (unit 3) }\end{array}$ & $\begin{array}{l}\text { sipo -- lingbao east interworking section } \\
\text { (unit 4) }\end{array}$ \\
\hline 3 & sipo tunnel (unit 5) & sipo tunnel (unit 6) \\
\hline 4 & sipo -yueliangwan section (unit 7) & yueliangwan- sipo section (unit 8)) \\
\hline 5 & yueliangwan and qiancun tunnel (unit 9) & yueliangwan and qiancun tunnel (unit 10) \\
\hline 6 & qiancun- maoyu section (unit 11) & maoyu— qiancun section (unit 12) \\
\hline 7 & mao yu tunnel (unit 13) & mao yu tunnel (unit 14) \\
\hline 8 & maoyu_lingxi section (unit 15) & maoyu—lingxi section (unit 16) \\
\hline 9 & lingxi tunnel (unit 17) & lingxi tunnel (unit 18) \\
\hline 10 & lingxi- lingnan section (unit 19) & lingxi- lingnan section (unit 20) \\
\hline 11 & lingnan tunnel (unit 21) & lingnan tunnel (unit 22) \\
\hline 12 & lingnan—zhoujiazui section (unit 23) & zhoujiazui_- lingnan section (unit 24) \\
\hline
\end{tabular}

\subsection{Traffic organization and control strategy}

When a tunnel of the tunnel group section had traffic accidents, we should carry out the traffic organization of the affected tunnel, and to follow the principle of "from adjacent to inside" and "from far to near" ${ }^{[5]}$. When the traffic incident occurred in a tunnel of Sipo to Lingnan tunnel group, the downstream vehicle of the accident tunnel can move on, while the upstream tunnels and setions to be limiting or disabling control, traffic control signal of the control and affected area to carry out the linkage control ${ }^{[6]}$.

Tunnel group section control strategy mainly has three ways, the mainline control、 the entrance ramp control and the road network traffic control. When the tunnel group section occurs serious traffic accidents, resulting in the large-scale traffic jam of the whole tunnel group, we should take distribution and current limiting measures to the outside section of the tunnel group ${ }^{[7]}$.

Assumed Sipo to Lingnan tunnel group's MaoYu tunnel right line occurs traffic accident, we must adopt linkage control to the tunnel group ${ }^{[8]}$.The left and right line's upstream section of the accident tunnel are the control area, and the upstream section of the control area is affected area, and the downstream section is no influence area, and the specific division of the control unit is shown in Figure 4 . 


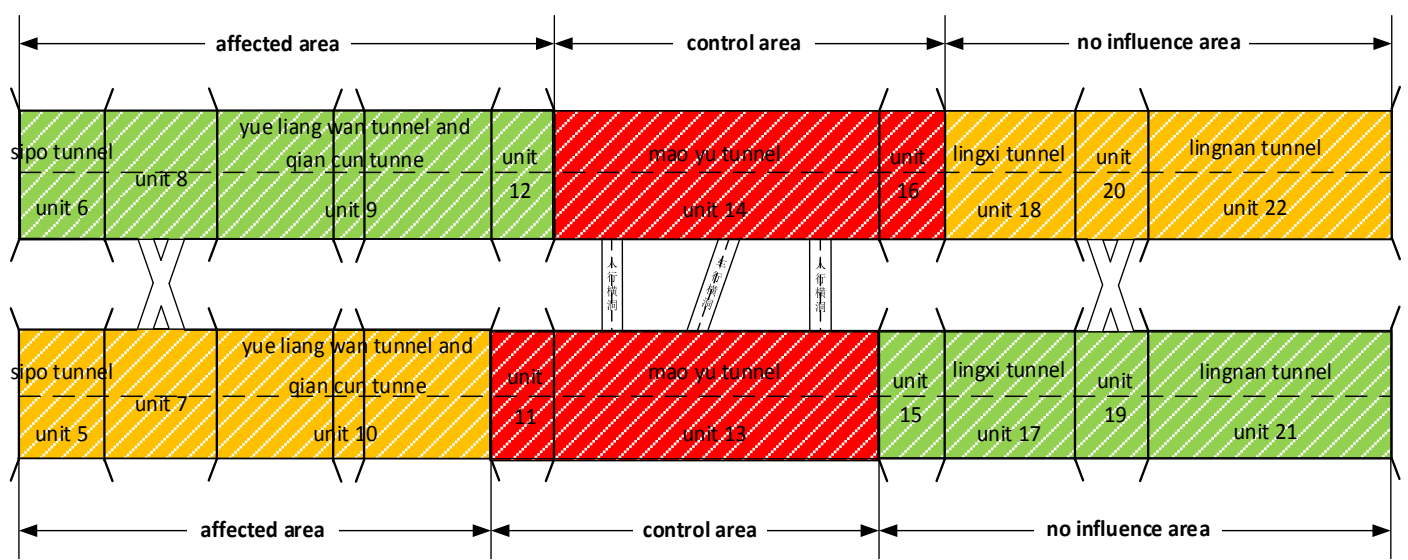

Fig. 4 The division of the control unit in the tunnel group The traffic organization control strategy is shown in figure 5 and figure 6 :

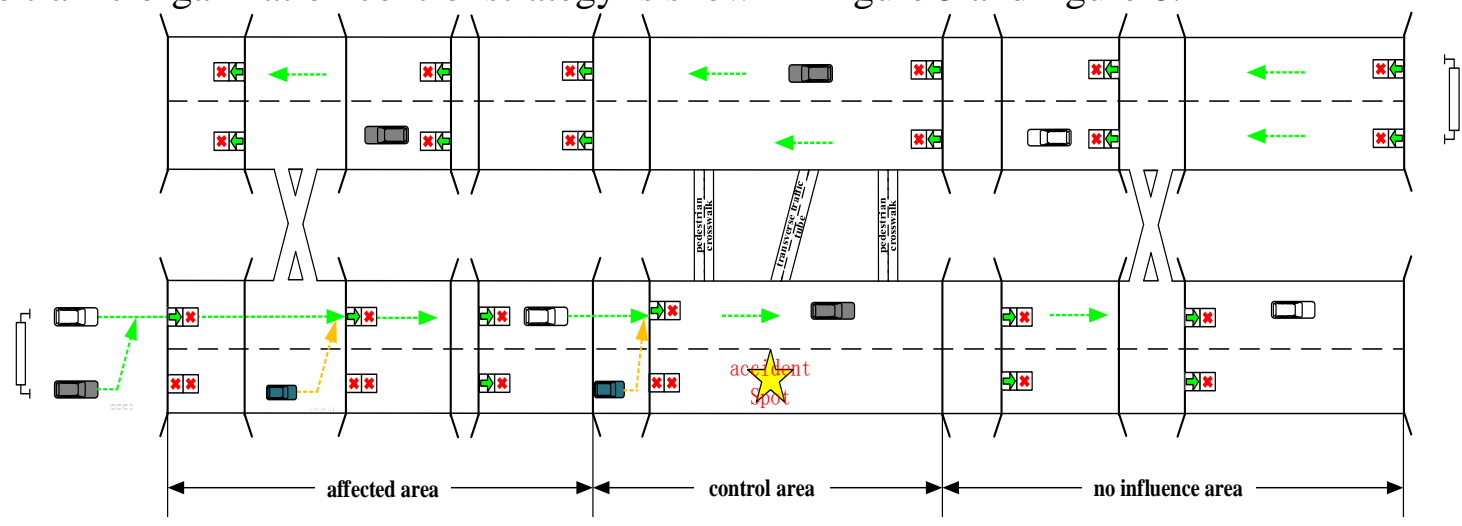

Fig. 5 Maoyu tunnel right line minor incident traffic organization and control strategy

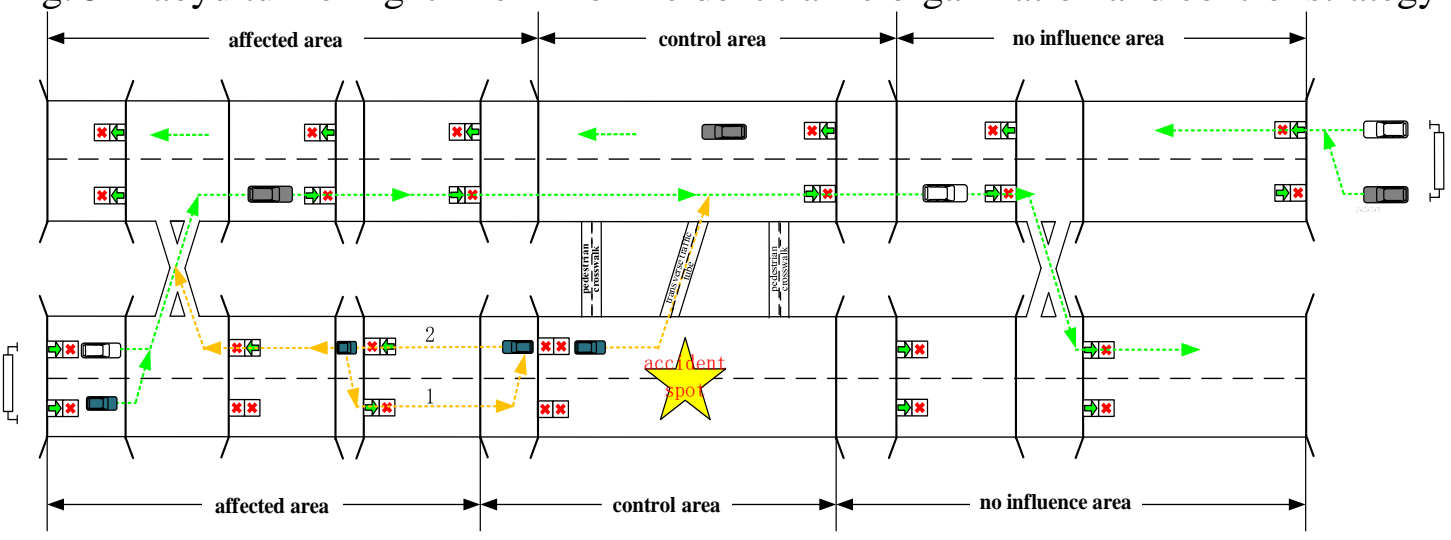

Fig. 6 Maoyu tunnel right line serious incident traffic organization and control strategy

\section{Tunnel linkage control strategy}

By Xiaoshan tunnel and Sipo to Lingnan tunnel group as the examples, the traffic organization and control strategy of the monomer tunnel and tunnel group is studied, the linkage control strategy are shown in Figure 7. 


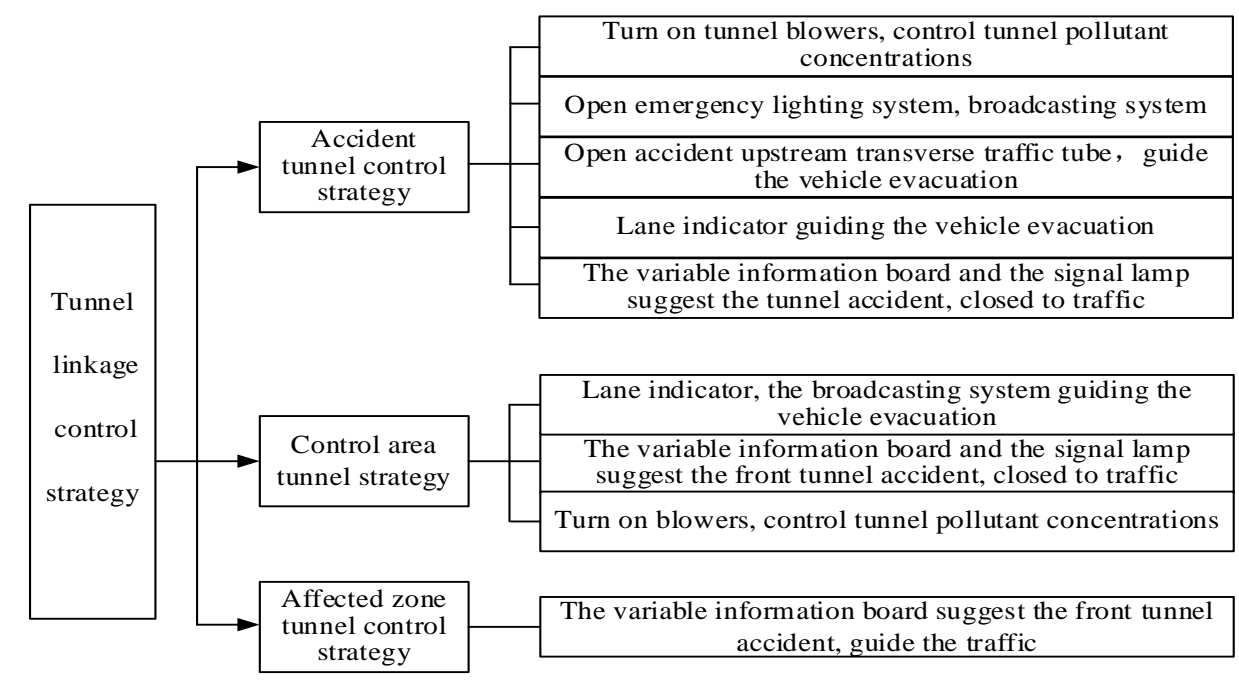

Fig. 7 Tunnel linkage control strategy

\section{Conclusion}

(1)Taking the actual operation expressway tunnel group as an example, investigation 、statistics and analysis three main factors that affect the traffic flow operating characteristics, the proportion of all types of vehicles v traffic volume change, and the driving speed of specific sections.

(2)Division the monomer tunnel of the prone or multiple tunnel group into control section, studied the monomer tunnel traffic organization and the control strategy in the case of a traffic accident.

(3) Division the prone or multiple tunnel group section into control unit, studied tunnel group's traffic organization and the control strategy in the case of a traffic accident.

(4) Through the engineering practice, summed up the tunnel linkage control strategy.

\section{References}

[1] National Bureau of Statistics of the People's Republic of China series. China statistical yearbook-2012[M]. Beijing: China Statistics Press

[2]Du Zhigang, Pan Xiaodong, Guo Bin, et al. Application Study on Safety Evaluation Index of Expressway Tunnel [J]. Journal of Tongji University, 2008，36(3)325-329

[3]Fang Yong, He Chuan, Li Haiying, et al. Study on Integrated and Coordinated Control Procedure and Scheme of Expressway TunnelGroups[A]. Technical Summary of Construction of Tunnel Groups on Wulong - Shuijiang Expressway in Chongqing. Beijing: China Communications Press, 2010, 198-204

[4]Guo Chun, Shi Hongqian, Wang Mingnian, et al. Study on Emergency Plan for Adjacent Tunnels and Tunnel Groups on Expressway under Fire Model [A]. Technical Summary of Construction of Tunnel Groups on Wulong Shuijiang Expressway in Chongqing. Beijing:China Communications Press, 2010, 205-208

[5]Liu Guiqiang, Wang Shaofei, Lu Hui, et al.Coordination Transportation Control Strategy of Interchange Road Section-Tunnel of Zeng-Cong Expressway [J]. Expressway, 2013, 03: 145-150

[6]Zhang Liyuan. Study on Safety Evaluation and Emergency Management Technology of Expressway Tunnel [D]. Chongqing Jiaotong University, 2011

[7]Zhang Jun. Study on Traffic Induction and Control Strategy of Expressway [D]. Chang'an University, 2008

[8]Zhang Anrui. Expressway Tunnel Group of Emergency Plan Formulation and Evaluation Technology Research [D]. Southwest Jiao Tong University, 2011 